Appl. Phys. B 48, 449-452 (1989)

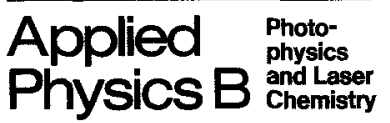

(C) Springer-Verlag 1989

\title{
Laser Performance of Perylenebis(Dicarboximide) Dyes with Long Secondary Alkyl Chains
}

\author{
H. G. Löhmannsröben ${ }^{1}$ and H. Langhals ${ }^{2}$ \\ ${ }^{1}$ Institut für Physikalische und Theoretische Chemie, Technische Universität, \\ Hans-Sommer-Strasse 10, D-3300 Braunschweig, Fed. Rep. Germany \\ ${ }^{2}$ Institut für Organische Chemie, Universität, Karlsstrasse 23, \\ D-8000 München 2, Fed. Rep. Germany
}

Received 9 January 1989/Accepted 16 February 1989

\begin{abstract}
The laser performance and related photophysical properties of two very soluble perylene dyes with long chain secondary alkyl groups were investigated in cyclohexane solution. With a dye laser as pump source a tuning range of $555-580 \mathrm{~nm}$ was obtained at an optimum concentration of $3 \times 10^{-4} \mathrm{M}$. The quantum efficiencies $(\eta=0.29$ and 0.21$)$ were better than $1 / 2$ that of rhodamine $6 \mathrm{G}$. No photodegradation was observed over an excitation period of several hours.
\end{abstract}

PACS: $42.55 \mathrm{M}, 82.50,33.20$

Among the polycyclic aromatic hydrocarbons $(\mathrm{PAH})$ perylene is distinguished by a high quantum yield of fluorescence $\left(Q_{\mathrm{F}}\right)$ and high stability. Naturally, perylene was one of the first molecules investigated as a potential laser dye. Early attempts with flashlamps as pump sources, however, failed to achieve lasing $[1,2]$. Later, laser action was demonstrated in laser-pumped arrangements but only with moderate conversion efficiencies and a small tuning range [3]. These shortcomings result mainly from a strong overlap of fluorescence emission and triplet absorption spectra. Many derivatives of perylene are available as dyestuffs of high quality [4]. They are epxected to be promising laser dyes if a reduction of the detrimental triplet absorption can be achieved. The perylene dyes, i.e. perylene-3,4:9,10-tetracarboxylic bisimides (PCI), are very photostable but generally have very low solubilities in organic solvents, which is prohibitive to applications in homogeneous solution. The solubility was increased by a substitution of the dyes with tert-butyl groups [5-7]. A significant further improvement was obtained by the attachment of long chain secondary alkyl groups to the perylene dye skeleton
[8]. Of these dyes the one with 1-hexylheptyl substituents ( $\mathbf{N}, \mathrm{N}^{\prime}$-bis(1-hexylheptyl) $\mathrm{PCI}$, dye 1) is readily soluble even in aliphatic hydrocarbons, and therefore was selected as a model compound for the investigation of the laser dye properties of PCI dyes. Laser action after dye laser excitation at ca. $500 \mathrm{~nm}$ and related photophysical properties were studied. For comparison, the corresponding properties of the $\mathrm{N}, \mathrm{N}^{\prime}$ bis(1-pentylhexyl) substituted PCI (dye 2) were also investigated.

The results of this work are related to two earlier studies of a tert-butylphenyl PCI derivative for which laser action [9] and excited state properties [10] have been reported. The present work extends the investigation of the laser performance of PCI dyes. The triplet state properties were found to be in general accordance with the results given in [10]. It has been noted before that the PCI dyes considered here exhibit spectroscopic features very similar to those of the PAH rubrene (5,6,11,12-tetraphenyltetracene) [10]. Rubrene can be considered as a suitable model compound for the lasing process in organic dyes [11]. Since the lasing potential of rubrene has recently been explored 
by one of us in some detail [11], a comparison with the dye laser characteristics of dye 1 can now be performed.

\section{Experimental}

A description of the experimental methods and of the equipment can be found in [11-13] and only details of the modified dye lasers will be given here. The dye laser used as pump source consisted of an excimer-pumped dye laser (Lambda Physik FL 1000) as oscillator stage which was extended by a home-built amplifier stage with $20 \mathrm{~mm}$ optical pathlength. With coumarine 307 (LC 5000) as laser dye, optimum output energies of $10 \mathrm{~mJ}$ with laser pulse durations of ca. $15 \mathrm{~ns}$ were obtained. As described in [11] this was used for transverse pumping of the dyes to be investigated in another simple dye laser. The latter had been modified in such a way that the usual $20 \mathrm{~mm}$ flow cells were replaced by a cuvette holder for standard $10 \times 10 \mathrm{~mm}$ optical cuvettes. Thus the investigation of small samples and their quick replacement was facilitated. The lack of circulation of the dye solutions prohibited repetition rates larger than $3 \mathrm{~Hz}$. All measurements were performed at room temperature and, if not noted otherwise, the solvent was spectroscopic grade cyclohexane. Laser action of dyes 1 and 2 was investigated in solutions under air, while for measurements of triplet state properties and of rubrene laser performance solutions were deoxygenated.

\section{Results and Discussion}

\subsection{Photophysical Properties of Dye 1}

The $\left(S_{0}-S_{1}\right)$ absorption spectrum of dye 1 shows that the dye is conveniently excited around $500 \mathrm{~nm}$ (molar extinction coefficient at $515 \mathrm{~nm}$ : $\varepsilon_{\mathrm{S}}(515 \mathrm{~nm})$ $=91200 \mathrm{M}^{-1} \mathrm{~cm}^{-1}$, Fig. 1). As opposed to that the

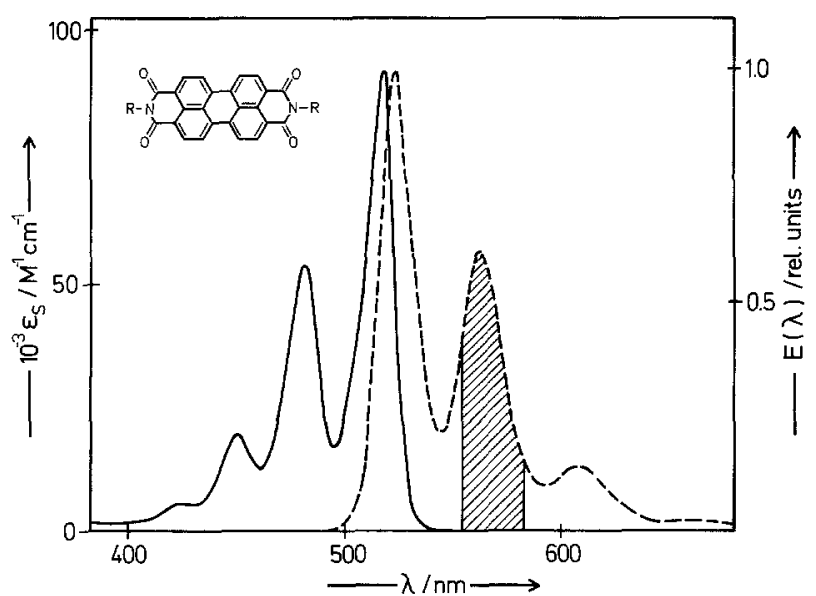

Fig. 1. Singlet absorption and fluorescence spectra of dye 1 ( $R=1$-hexylheptyl) in cyclohexane. The hatched area denotes the laser tuning range absorbance in the near UV region is small $\left[\varepsilon_{\mathrm{S}}(308 \mathrm{~nm})\right.$ $\left.\approx \varepsilon_{\mathrm{S}}(337 \mathrm{~nm}) \leqq 4500 \mathrm{M}^{-1} \mathrm{~cm}^{-1}\right]$ and attempts to observe laser action after nitrogen laser excitation have not been rewarding $[9,14]$. The fluorescence lifetime of dye 1 was measured to be $\tau_{\mathrm{F}}=5.8 \pm 0.2 \mathrm{~ns}$ and within experimental accuracy $Q_{F}$ is unity. An important indication for the quality of laser dyes is the crosssection of fluorescence emission $\left(\sigma_{\mathrm{e}}\right)$ as obtained from [15]:

$\sigma_{\mathrm{e}}=\lambda^{4} E(\lambda) Q_{\mathrm{F}} /\left(8 \pi \tau_{\mathrm{F}} c n^{2}\right)$.

Here $E(\lambda)$ is the normalized fluorescence lineshape function, $n$ the refractive index of the solution and $c$ the velocity of light. For dye 1 a value of $\sigma_{\mathrm{e}}=1.7$ $\times 10^{-16} \mathrm{~cm}^{2}$ was obtained at $564 \mathrm{~nm}$ which rivals the values found for the best laser dyes (e.g. for rhodamine $6 \mathrm{G} \sigma_{\mathrm{e}}=2.2 \times 10^{-16} \mathrm{~cm}^{2}$ at $\left.565 \mathrm{~nm}[15]\right)$.

Triplet state properties of dye 1 were studied by the energy transfer method [16] with anthracene as triplet sensitizer. The recorded change of absorbance $\Delta A$, which is proportional to the difference of the extinction coefficients of triplet and singlet absorption $\left(\varepsilon_{\mathrm{T}}-\varepsilon_{\mathrm{S}}\right)$, and the triplet absorption spectrum are shown in Fig. 2. The triplet lifetime of dye 1 was measured to be: $\tau_{\mathrm{T}}=(2.8 \pm 0.6) \times 10^{-4} \mathrm{~s}$. Since both our investigation of dye 2 and the data in [10] gave similar results these triplet state properties can be considered as typical for many PCI dyes. With respect to the laser potential of these dyes it is of particular importance that they show only a small overlap of triplet absorption and fluorescence spectra (Fig. 1).

\subsection{Laser Dye Performance of Dye 1}

With dye laser pumping between $480-510 \mathrm{~nm}$ laser action of $(1-5) \times 10^{-4} \mathrm{M}$ solutions of dye 1 was readily achieved. Optimum energy output was obtained at a concentration of $3 \times 10^{-4} \mathrm{M}$ with a tuning range of $555-580 \mathrm{~nm}$ (Fig. 3). Optimum concentration and tuning range of dye 2 were almost identical. No degradation of $3 \mathrm{ml}$ dye solutions was noticed over an excitation period of $6 \mathrm{~h}(3 \mathrm{~Hz}$ repetition rate, pump laser energy ca. $7.5 \mathrm{~mJ}$ with a beam diameter of $3 \mathrm{~mm}$ ). Since even at significantly lower concentrations no lasing occurred below $550 \mathrm{~nm}$ the spectral region of maximum $\sigma_{\mathrm{e}}$ remained excluded from laser emission. This reflects the effect of singlet absorption in this region (Fig. 3) which results from the small Stokes shift in dye 1 (Fig. 1). As a measure of the laser dye quality [17] the quantum efficiencies $\eta$ of dyes 1 and 2 were determined from plots of dye laser energy outputs $\left(E_{\text {Dye }}\right)$ vs. pump laser energy $\left(E_{\text {exc }}\right)$ as presented in Fig. 4. For comparison, $\eta$ of rhodamine $6 \mathrm{G}$ (Rh $6 \mathrm{G}$, in methanol) was also measured under identical conditions. The values obtained in the maxima of the 


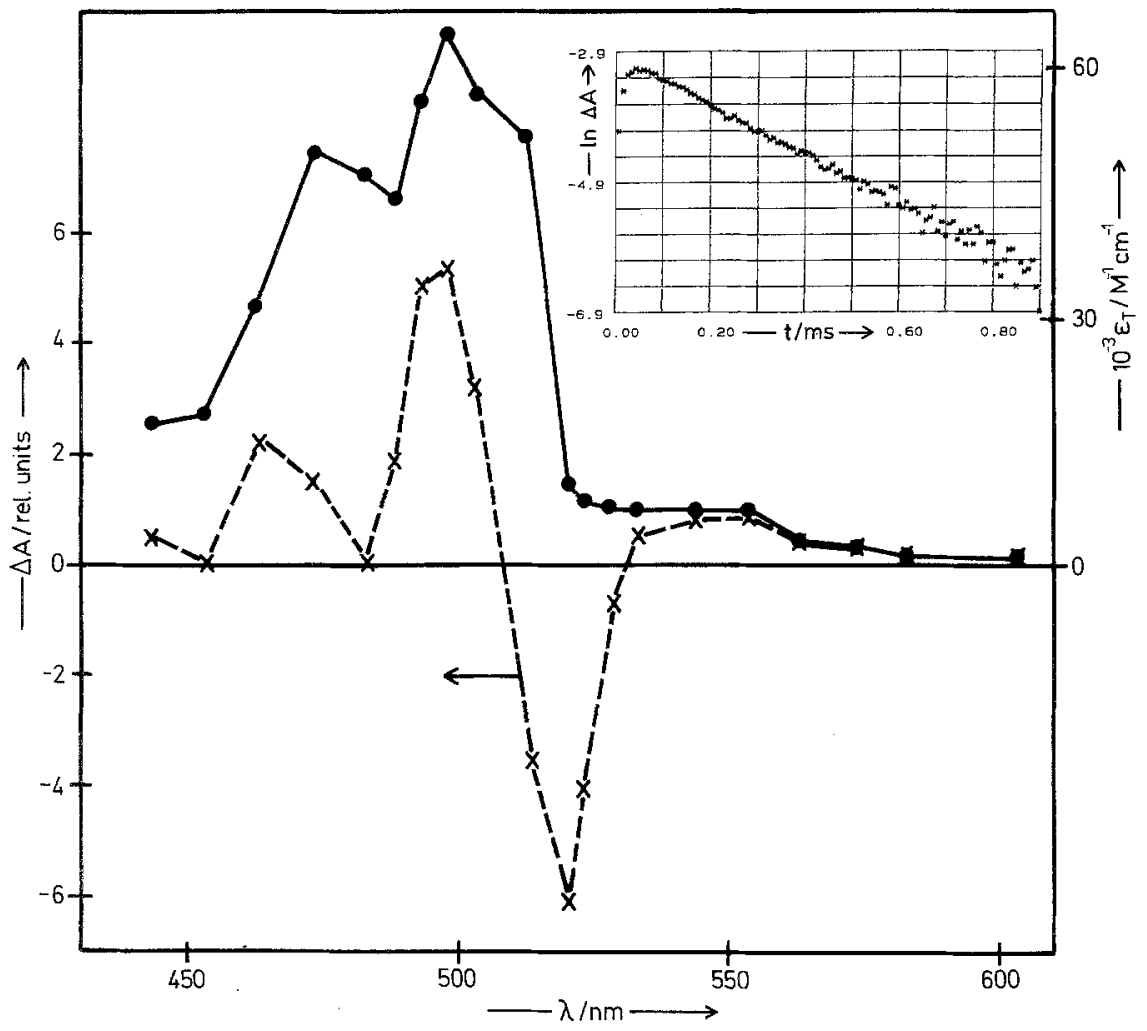

Fig. 2. Recorded change of absorbance $\Delta A$ after triplet sensitization with anthracene and triplet absorption spectrum of dye 1. The insert shows the decay of triplet molecules monitored at $495 \mathrm{~nm}$

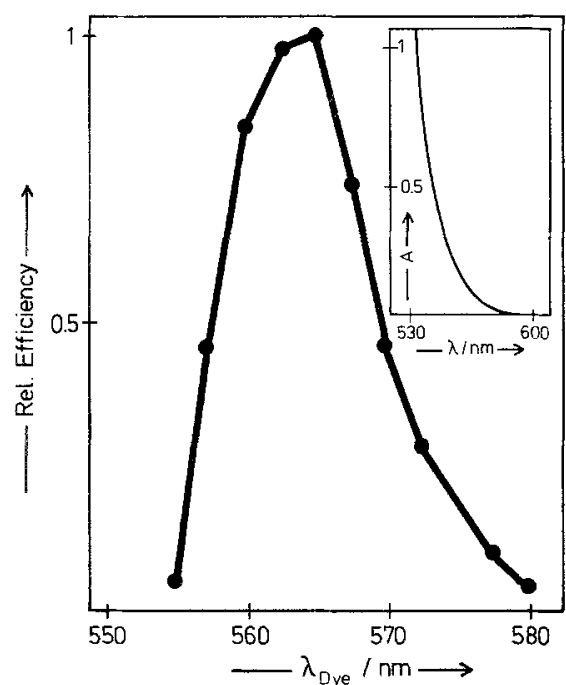

Fig. 3. Laser tuning range of a $3 \times 10^{-4} \mathrm{M}$ solution of dye 1 in cyclohexane. The insert shows the absorbance of that solution in the spectral range of fluorescence emission

tuning ranges were: $\eta=0.29$ at $564 \mathrm{~nm}$ for dye 1 , $\eta=0.21$ at $562 \mathrm{~nm}$ for dye 2 and $\eta=0.40$ at $565 \mathrm{~nm}$ for Rh 6G. In an earlier work with $308 \mathrm{~nm}$ excitation a quantum efficiency of 0.09 at $565 \mathrm{~nm}$ was found for rubrene [11].

The evaluation of these results supports the following conclusions about the lasing potential of the PCI

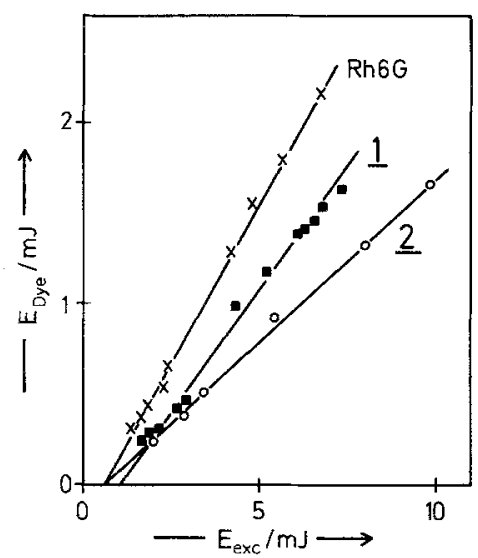

Fig. 4. Dye laser output with PCI dyes 1 (at $564 \mathrm{~nm}$ ) and 2 $(562 \mathrm{~nm})$ and with rhodamine $6 \mathrm{G}(565 \mathrm{~nm}$, in methanol) vs. pump laser energy

dyes investigated here: Both dyes 1 and 2 are very stable dyes with quantum efficiencies less than a factor of two below that of Rh $6 \mathrm{G}$, one of the most successful commercial dye. They significantly surpass the efficiency and stability of rubrene. The tuning range of the PCI dyes, however, is significantly smaller than that of good dyes in the spectral region of interest. Lasing of 
rubrene, for example, is now continuously tunable from $554-605 \mathrm{~nm}$. Further synthetic effort is necessary to provide PCI dyes with larger Stokes shift. If lasing could be achieved in the spectral region of strongest fluorescence not only the tuning range but also the quantum efficiency would improve.

\section{References}

1. D.L. Stockman, W.R. Mallory, F.K. Tittel: Proc. IEEE 52, 318 (1964)

2. P.P. Sorokin, J.R. Lankard, V.L. Moruzzi, E.C. Hammond: J. Chem. Phys. 48, 4726 (1968)

3. Cl. Rulliere, M. Laughrea, M.M. Denariez-Roberge: Opt. Commun. 6, 407 (1972)

4. H. Zollinger: Color Chemistry (VCH, Weinheim 1987)

5. H. Langhals: Chem. Ber. 118, 4641 (1985)
6. H. Langhals: D.O.S. 3016764 (30.4.1980); Chem. Abstr. 96, (1982) $70417 \mathrm{x}$

7. A. Rademacher, S. Märkle, H. Langhals: Chem. Ber. 115, 2927 (1982)

8. S. Demming, H. Langhals: Chem. Ber. 121, 225 (1988)

9. M. Sadrai, G.R. Bird: Opt. Commun. 51, 62 (1984)

10. W.E. Ford, P.V. Kamat: J. Phys. Chem. 91, 6373 (1987)

11. H.-G. Löhmannsröben: Appl. Phys. B 47, 195 (1988)

12. F. Lewitzka, H.-G. Löhmannsröben: Z. Phys. Chem. NF 150, 69 (1986)

13. C. Burgdorff, T. Kircher, H.-G. Löhmannsröben: Spectrochim. Acta 44 A, 1137 (1988)

14. E.M. Ebeid, S.A. El-Daly, H. Langhals: J. Phys. Chem. 92, 4565 (1988)

15. L.G. Nair: Progr. Quant. Electron. 7, 153 (1982)

16. I. Carmichael, G.L. Hug: J. Phys. Chem. Ref. Data 15, 1 (1986)

17. G. Marowsky: Opt. Acta 23, 855 (1976) 